\title{
Éclosion de rougeole au sein d'une population non vaccinée, Colombie-Britannique,
} 2014

\author{
Naus $M^{1,2 *}$, Puddicombe $D^{1}$, Murti $M^{3}$, Fung $C^{3}$, Stam $R^{3}$, Loadman $S^{3}$, Krajden $M^{1,2}$, Tang $P^{1,2}$, Lem $M^{2,3}$ \\ ${ }^{1}$ Centre de contrôle des maladies de la Colombie-Britannique, Vancouver (Colombie-Britannique) \\ ${ }^{2}$ Faculté de médecine, Université de la Colombie-Britannique, Vancouver (Colombie-Britannique) \\ ${ }^{3}$ Fraser Health Authority, Surrey (Colombie-Britannique) \\ *Correspondance : monika.naus@bccdc.ca
}

\section{Résumé}

Contexte : Bien que le Canada ait éliminé la rougeole endémique en 1998, on s'attend à ce que des éclosions résultant de la transmission par un cas importé surviennent de temps à autre au sein de communautés non vaccinées et regroupées géographiquement. Au printemps 2014, une importante éclosion de rougeole liée à une éclosion aux Pays-Bas est survenue en Colombie-Britannique dans une communauté non vaccinée pour des raisons religieuses.

Méthodologie : Une recherche des cas a été réalisée avec l'aide de la communauté locale, de ses écoles et de ses dirigeants de groupes religieux ainsi que des fournisseurs de soins de santé locaux pour dénombrer les cas confirmés, probables et suspects. Des lignes directrices pour le contrôle de la rougeole ont été mises en œuvre avec une acceptation limitée du vaccin contenant le virus de la rougeole, mais une observance accrue des mesures de contrôle des infections et des restrictions de voyage.

Résultats : Au total, 433 cas (325 cas confirmés et 108 cas probables) ont été détectés. L'apparition de l'éruption cutanée s'est échelonnée du 22 février au 9 juin, et pendant les mois de mars et d'avril dans $98 \%$ des cas. Cinquante-sept pour cent des cas sont survenus chez les élèves d'une école. L'âge médian des cas était de 11 ans et $68 \%$ des cas sont survenus chez des personnes de 5 à 19 ans. Quatre-vingt-dix-neuf pour cent des cas sont survenus chez des personnes non vaccinées. Une personne a souffert d'encéphalite et s'est rétablie. Seulement cinq cas sont survenus en dehors de la communauté touchée. Les résultats du génotypage indiquent une importation de l'éclosion aux Pays-Bas.

Conclusion : cette éclosion dans une communauté où le taux de vaccination est faible a touché en grande partie la population pédiatrique, les adultes ayant probablement acquis l'immunité contre la rougeole à la suite d'une infection antérieure par le virus de la rougeole de type sauvage. Malgré l'hésitation persistante de cette population à se faire vacciner, la collaboration élevée de la communauté pour respecter les mesures de contrôle de l'infection et les restrictions de déplacement a facilité l'endiguement de l'éclosion.

\section{Introduction}

La rougeole est une maladie virale aiguë hautement contagieuse et évitable grâce au vaccin contre la rougeole. La Colombie-Britannique (C.-B.) a lancé en 1996 un programme de vaccination des enfants en deux doses $(1,2)$ en vue d'atteindre l'objectif de l'Organisation panaméricaine de la santé (OPS) d'éliminer la rougeole. Le Canada a éliminé la rougeole endémique (définie comme étant issue d'une chaîne de transmission identifiable qui dure au moins 12 mois) depuis 1998, et l'élimination de la rougeole dans les Amériques a été déclarée en 2002. L'état d'élimination de la rougeole a récemment fait l'objet d'une révision par le comité international d'experts (3).

Des cas et des éclosions de rougeole continuent de survenir au Canada en raison d'une importation périodique. Depuis que le Canada a éliminé la rougeole endémique, la C.-B. a été le théâtre de deux éclosions importantes. La première éclosion, en 2010, a entraîné 82 cas confirmés et cliniques (également appelés " probables "), s'est propagée à l'échelle de la province et a été associée à deux importations distinctes pendant les Jeux olympiques 
d'hiver de Vancouver $(4,5)$. La deuxième éclosion, nettement plus importante, est survenue en 2014 et est décrite dans le présent relevé.

L'éclosion de 2014 a eu lieu dans la région sanitaire de l'est de la vallée du Fraser dans une communauté protestante orthodoxe réformée des Pays-Bas dont la population est estimée à 1200 personnes. Cette communauté est reconnue comme étant opposée à la vaccination. Sa population, qui n'est pas isolée socialement ni géographiquement, réside dans un secteur agricole semi-rural de la région et entretient des liens étroits avec les Pays-Bas. L'éclosion est survenue à la toute fin d'une éclosion de rougeole qui a eu lieu aux Pays-Bas de mai 2013 à mars 2014. L'éclosion des Pays-Bas a entraîné près de 2600 cas déclarés de rougeole, 182 hospitalisations d'enfants et le décès d'un enfant attribuable à des complications de la rougeole $(6,7)$. La même éclosion aux Pays-Bas a entraîné deux importations antérieures dans la région de la Fraser Health Authority, en août et en novembre 2013, sans transmission de la maladie à grande échelle, et une importation de la rougeole dans le sud de l'Alberta qui a donné lieu à 42 cas confirmés d'octobre 2013 à janvier 2014 (8).

\section{Enquête sur l'éclosion}

Le 6 mars 2014, la Fraser Health Authority a été avisée par le directeur d'une école de l'est de la région qu'environ le tiers d'une classe d'élèves de troisième année et environ $10 \%$ des autres élèves s'étaient absentés de l'école en raison d'une maladie qui s'accompagne d'une éruption cutanée. Presque tous les élèves de l'école n'avaient pas été vaccinés contre la rougeole en raison de croyances religieuses.

Le bureau de santé a amorcé les démarches de confirmation et de recherche des cas en téléphonant aux familles des enfants de l'école pour dénombrer les cas antérieurs et actuels. Les familles de l'école étaient généralement nombreuses et étroitement liées par l'intermédiaire de l'école, de l'église et de relations avec la famille élargie. La plupart des familles comptant un ou plusieurs enfants malades n'avaient pas sollicité de soins médicaux et ont refusé l'évaluation médicale et les analyses de laboratoire.

\section{Recherche des cas et activités de collecte de données}

Conformément aux règlements sur les maladies contagieuses de la Loi sur la santé publique en ColombieBritannique (9), les cas confirmés, probables et suspects de rougeole doivent être déclarés. La définition de cas provinciale de la rougeole a été utilisée pour le classement des cas, mais modifiée pour inclure les cas au « lien épidémiologique confirmé », p. ex. les élèves et les membres du personnel de l'école touchée qui présentaient une maladie clinique compatible avec la rougeole (10). Cette modification a été fondée sur l'hypothèse que la fréquentation de l'école a entraîné une exposition à la rougeole comparable à une exposition à un cas confirmé en laboratoire.

Un formulaire de déclaration de cas modifié et abrégé inspiré du formulaire provincial pour les cas de rougeole a été utilisé pendant l'éclosion et présenté au Centre de contrôle des maladies de la Colombie-Britannique pour la saisie des données. Le formulaire de déclaration de cas provincial a été présenté pour chaque cas à la fin de l'éclosion (11). Les données démographiques, les signes et symptômes, les antécédents de vaccination, les complications et les résultats, les échantillons de laboratoire recueillis, les antécédents de voyage et d'exposition, une profession dans le domaine de la santé, la fréquentation d'un établissement d'enseignement, la résidence dans un milieu communautaire et des données agrégées sur les contacts faisaient partie des variables.

\section{Méthodes de laboratoire}

Le Laboratoire de santé publique et de référence en microbiologie de la Colombie-Britannique a réalisé les tests moléculaires de dépistage de l'ARN viral sur des écouvillonnages du nasopharynx et des échantillons d'urine par transcription inverse suivie d'une réaction en chaîne de la polymérase en temps réel (RT-PCR). Le dépistage sérologique a été réalisé par immuno-essai au moyen des tests Enzygnost@ Anticorps anti-Rougeole/lgG et Anticorps anti-Rougeole/lgM de Siemens. Le Laboratoire national de microbiologie (LNM) de Winnipeg a réalisé les tests RT-PCR de confirmation et le génotypage du virus pour tous les échantillons positifs à la suite des tests RT-PCR. 


\section{Mesures de santé publique}

Compte tenu du risque de propagation élevé de la rougeole en provenance des Pays-Bas à la population associée en Colombie-Britannique, les bureaux locaux de santé publique de la Fraser Health Authority ont déployé des efforts concertés avant cette éclosion à l'été 2013 pour renforcer les relations avec les communautés néerlandaises réformées, en particulier celles dont les écoles sont administrées en collaboration avec les églises. Ces démarches ont été réalisées avec l'aide d'une infirmière membre de la communauté néerlandaise réformée embauchée par la Fraser Health Authority pour agir en tant que ressource communautaire. Par ailleurs, des conseils ont été sollicités auprès de professionnels de la santé publique de la région du sud de l'Alberta où vivent des communautés semblables. Puisque la plupart des membres de la communauté allaient refuser le vaccin contre la rougeole malgré l'éclosion, la discussion entre les autorités sanitaires et la communauté a été orientée sur des valeurs et des objectifs communs. Les valeurs qui ont été abordées sont la responsabilité partagée du bien-être de la communauté, la nécessité de protéger les membres vulnérables de la société et la volonté de ne causer aucun tort. La déclaration rapide des cas, la minimisation de la transmission en dehors de la communauté et l'optimisation des mesures de contrôle des infections sont les objectifs qui ont été fixés.

La gestion des cas et des contacts a été assurée conformément aux lignes directrices pour le contrôle de la rougeole de la Colombie-Britannique (12). La prophylaxie post-exposition au moyen du vaccin contre la rougeole, les oreillons et la rubéole (ROR) ainsi que d'immunoglobulines (IG) a été offerte. Presque tous les membres de la communauté religieuse ont refusé le vaccin, mais l'administration des IG a été jugée conforme à la doctrine religieuse puisque la communauté la considérait comme un « traitement ». Les membres de la communauté consentant à la vaccination ont pu obtenir le service de façon confidentielle pour éviter d'être ostracisés par leur groupe confessionnel. Les personnes infectées ont reçu la consigne de s'isoler pendant la phase prodromique et les quatre jours suivant l'apparition de l'éruption cutanée et les personnes exposées, de se soumettre à la quarantaine pendant une période de 21 jours. Des ententes ont été conclues avec les laboratoires des hôpitaux pour obtenir des services de diagnostic par l'entremise d'un mode de paiement de rechange pour les membres de la communauté qui ne détenaient pas de numéro de régime d'assurance médicale. Les infirmières en santé publique n'ont pas incité la population à subir des analyses de laboratoire après la confirmation de l'éclosion pour éviter l'exposition du grand public, par exemple dans les salles d'attente, après une exposition potentielle dans les milieux de soins locaux.

La Fraser Health Authority a rapidement déployé des mesures de contrôle dans la région de la vallée du Fraser et a collaboré avec de nombreux groupes au sein de la communauté touchée, y compris les écoles et les dirigeants des groupes religieux. Comme l'éclosion a commencé une semaine avant la semaine de relâche, on a demandé aux familles des personnes infectées ou ayant été exposées à la maladie de ne pas voyager. La commission scolaire a consenti à ces restrictions de voyage et les dirigeants de la communauté les ont appuyées. Le directeur a fermé l'école le 7 mars, deux jours avant le début de la semaine de relâche et, conformément aux directives du médecin hygiéniste en chef, l'école est restée fermée pendant une autre semaine par la suite, jusqu'au $1^{\mathrm{er}}$ avril.

La Fraser Health Authority a également recommandé aux résidents de la région non vaccinés ou présentant une couverture vaccinale insuffisante de recevoir le vaccin contre la rougeole au moyen d'une variété de communications et de services de vaccination qui ont été offerts par les autorités sanitaires ainsi que dans les pharmacies et les cabinets de médecins locaux. Une deuxième dose du vaccin ROR a été offerte aux tout-petits des membres de la population environnante qui ont accepté la vaccination dès le premier mois suivant la première dose (autrement, le calendrier de vaccination régulier prévoit une deuxième dose vers l'âge de quatre à six ans). Une preuve d'immunisation contre la rougeole était exigée de la part de tous les membres du personnel de la Fraser Health Authority pour travailler dans un milieu de soins aux patients. L'éclosion a été déclarée le 29 avril.

\section{Analyse statistique}

Les statistiques descriptives ont été calculées au moyen de Stata 13 (StataCorp. [2013]. Logiciel statistique Stata : version 13. College Station, TX : StataCorp LP). 


\section{Résultats}

Au total, 433 cas ( 325 confirmés et 108 probables) ont été dénombrés pendant l'éclosion parmi les résidents de la C-B. L'éruption cutanée du cas index est apparue le 22 février 2014 (semaine épidémiologique 8). Les milieux communs où il a été établi que la transmission de ce cas a eu lieu sont l'église pendant la deuxième journée de l'éruption cutanée et l'école pendant la troisième journée. L'éruption cutanée est survenue en mars et en avril (semaines épidémiologiques 10 à 16) dans la plupart des cas (425, $98 \%$ ) et le 9 juin 2014 (semaine épidémiologique 24) dans le dernier cas (Figure 1).

Figure 1: Courbe épidémique de l'éclosion de rougeole par état des cas et par semaine épidémiologique d'apparition de l'éruption cutanée, Colombie-Britannique, 2014

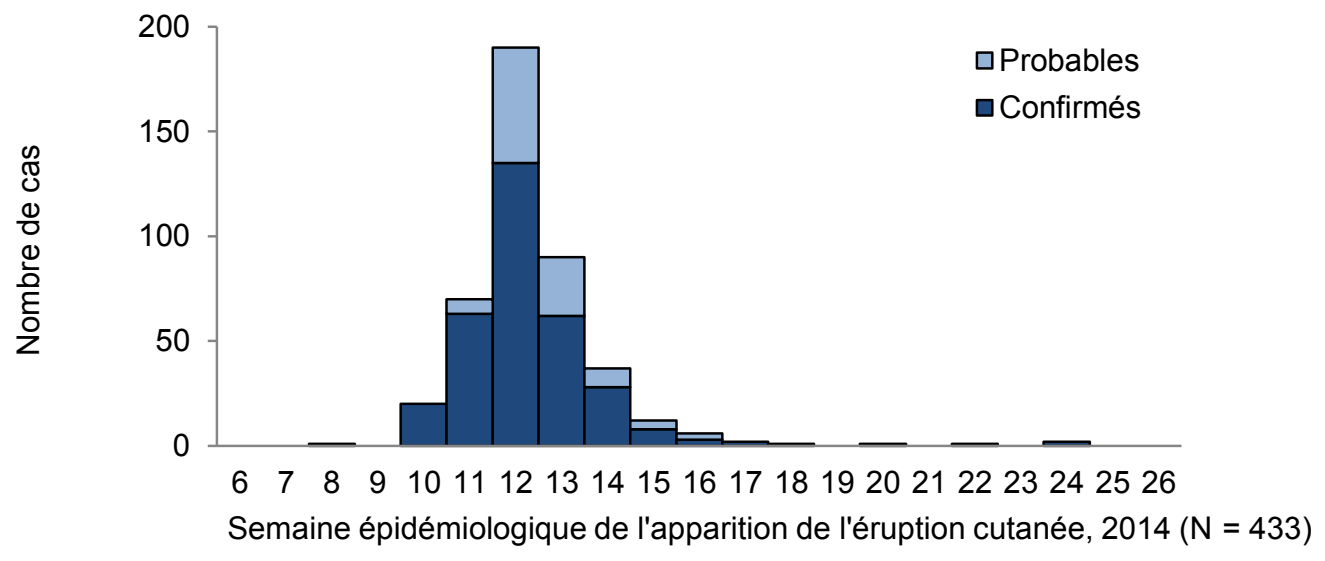

L'éclosion était concentrée dans une école de la région qui comptait environ 360 élèves de la maternelle à la $12^{\mathrm{e}}$ année. Dans plus de la moitié des cas, les personnes infectées étaient des élèves ou des membres du personnel de l'école (248 cas, 57 \%; 13 confirmés en laboratoire et 235 présentant un lien épidémiologique).

Trente-trois ( $8 \%$ ) cas confirmés en laboratoire (29 par test de polymérase en chaîne et 4 par test sérologique d'lgM), 108 (25\%) cas probables et $292(67 \%)$ cas présentant un lien épidémiologique ont été dénombrés (Tableau 1). La répartition des cas selon l'âge est présentée dans le Tableau 1. L'âge médian était de 11 ans. Parmi les 433 cas, seulement 9 (2 pour cent) personnes avaient moins de 1 an, 70 (16\%) avaient de 1 à 4 ans, $295(68 \%)$ étaient des enfants d'âge scolaire de 5 à 19 ans (14 \%) et $59(14 \%)$ avaient de 20 à 44 ans. Les cas confirmés concernaient moins souvent des personnes de moins de 5 ans que des personnes de 5 à 19 ans (Figure 2), un résultat probablement attribuable à la composante de fréquentation scolaire dans la définition du lien épidémiologique. Cinquante et un pour cent des cas sont survenus chez des hommes. 
Tableau 1: Caractéristiques des cas de l'éclosion de rougeole par état des cas, ColombieBritannique, 2014

\begin{tabular}{|c|c|c|c|c|c|c|}
\hline \multirow[t]{2}{*}{ Caractéristique } & \multicolumn{2}{|c|}{$\begin{array}{l}\text { Cas confirmés } \\
\qquad \mathbf{N}=325\end{array}$} & \multicolumn{2}{|c|}{$\begin{array}{l}\text { Cas probables } \\
\qquad \mathrm{N}=108\end{array}$} & \multicolumn{2}{|c|}{$\begin{array}{c}\text { Tous les cas } \\
\mathrm{N}=433\end{array}$} \\
\hline & $\mathbf{n}$ & $\%$ & $\mathbf{n}$ & $\%$ & 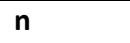 & $\%$ \\
\hline \multicolumn{7}{|l|}{ Classification des cas } \\
\hline $\begin{array}{l}\text { Confirmés par test de } \\
\text { polymérase en chaîne }\end{array}$ & 29 & - & - & - & 29 & 7 \\
\hline $\begin{array}{l}\text { Confirmés par test de détection } \\
\text { d'IgM }\end{array}$ & 4 & - & - & - & 4 & 1 \\
\hline $\begin{array}{l}\text { Présentant un lien } \\
\text { épidémiologique confirmé }\end{array}$ & 292 & - & - & - & 292 & 67 \\
\hline Probables & - & - & 108 & - & 108 & 25 \\
\hline \multicolumn{7}{|l|}{ Groupe d'âge (ans) } \\
\hline$<1$ & 2 & 1 & 7 & 6 & 9 & 2 \\
\hline 1 à 4 & 16 & 5 & 54 & 50 & 70 & 16 \\
\hline 5 à 9 & 115 & 35 & 4 & 4 & 119 & 27 \\
\hline 10 à 19 & 161 & 50 & 15 & 14 & 176 & 41 \\
\hline 20 à 44 & 31 & 10 & 28 & 26 & 59 & 14 \\
\hline \multicolumn{7}{|l|}{ Sexe } \\
\hline Hommes & 166 & 51 & 56 & 52 & 222 & 51 \\
\hline Femmes & 159 & 49 & 52 & 48 & 211 & 49 \\
\hline \multicolumn{7}{|l|}{ Objection à la vaccination } \\
\hline Non & 16 & 5 & 3 & 3 & 19 & 4 \\
\hline Oui & 266 & 82 & 90 & 83 & 356 & 82 \\
\hline Inconnue & 43 & 13 & 15 & 14 & 58 & 13 \\
\hline \multicolumn{7}{|l|}{ Antécédents vaccinaux } \\
\hline 0 dose & 281 & 86 & 93 & 86 & 374 & 86 \\
\hline 1 dose documentée & 1 & 0 & 2 & 2 & 3 & 1 \\
\hline
\end{tabular}




\begin{tabular}{|l|r|r|r|r|r|r|}
\hline 2 doses documentées & 1 & 0 & 1 & 1 & 2 & 0 \\
\hline Inconnus & 42 & 13 & 12 & 11 & & 54 \\
\end{tabular}

"Un résident de l'état de Washington a contracté la rougeole après y avoir été exposé dans la région de l'est de Fraser et a été la source d'infection de cinq autres cas déclarés dans l'état de Washington. Ces six cas ne font pas partie des 433 cas décrits dans les présentes.

Figure 2 : Nombre de cas par groupe d'âge et par état de cas

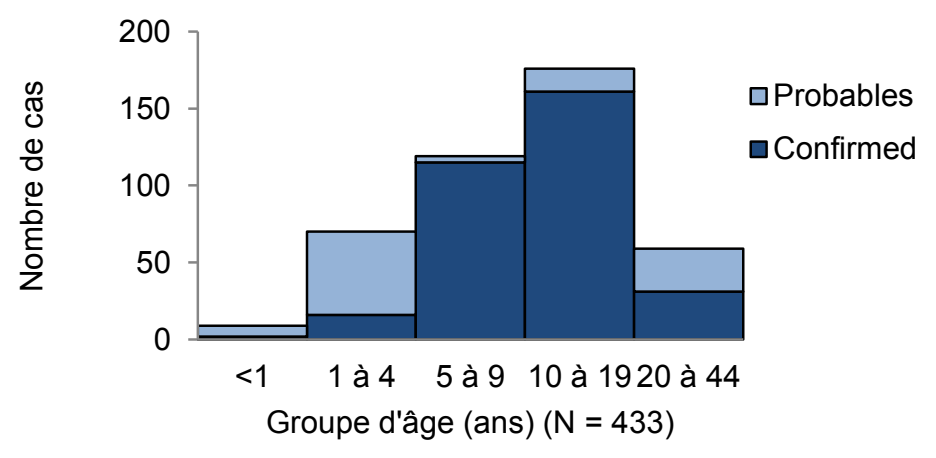

Dans la plupart des cas (356 cas, $82 \%$ ), une objection à la vaccination a été soulevée, et aucune objection n'a été soulevée dans seulement 19 cas $(4 \%)$. L'objection à la vaccination n'a pas été rapportée dans 58 cas (13\%). Au moins une dose du vaccin contenant le virus de la rougeole a été reçue antérieurement dans seulement cinq cas (un pour cent). La répartition selon le sexe et la vaccination était semblable pour les cas confirmés et les cas probables. Aucun voyage pendant la période d'exposition n'a été signalé parmi les cas liés à l'éclosion. Trois cas ont présenté une pneumonie et un cas, des convulsions fébriles. Une autre personne a souffert d'encéphalite et s'est rétablie. On dénombre cinq hospitalisations et aucun décès. Seulement cinq cas ont été repérés chez des personnes qui n'étaient pas membres de la communauté religieuse : trois cas au sein d'une famille comptant deux enfants non vaccinés et deux cas qui ont déclaré avoir reçu deux doses du vaccin ROR. La propagation en dehors de la communauté religieuse a été contenue grâce à des taux relativement élevés de vaccination contre la rougeole. Dans l'ensemble, $90 \%$ des enfants de 7 ans de la région ont reçu les deux doses du vaccin contenant le virus de la rougeole en 2014.

Le génotype D8 a été identifié dans 28 cas de rougeole confirmés par test de polymérase en chaîne. Parmi ces cas, 27 étaient totalement identiques à la variante de la séquence MVs/Taunton.GBR/27.12 (la même que la souche associée à l'éclosion aux Pays-Bas) et un y était identique à 99,8\%.

\section{Discussion}

Cette éclosion importante survenue en 2014 dans une communauté confessionnelle reconnue pour avoir un faible taux de vaccination a touché en grande partie la population pédiatrique, les adultes ayant probablement acquis l'immunité contre la rougeole à la suite d'une infection antérieure par le virus de la rougeole de type sauvage. L'épicentre était une école, ce qui a favorisé une transmission rapide et intense. Malgré l'hésitation persistante de cette population à se faire vacciner, la collaboration élevée de la communauté pour respecter les mesures de contrôle de l'infection et les restrictions de déplacement a facilité l'endiguement de l'éclosion.

Bien que 433 cas aient été signalés, le nombre véritable de cas est probablement plus élevé puisque cette communauté n'a pas tendance à solliciter des diagnostics et des traitements médicaux. De nombreux membres de cette communauté ne souscrivent pas au régime de soins de santé provincial et ne peuvent pas faire l'objet 
d'un suivi dans les bases de données administratives. L'éclosion a été limitée à la communauté affectée et a entraîné seulement cinq cas chez des personnes qui n'en sont pas membres malgré des situations se prêtant à une transmission potentielle, notamment dans les transports en commun, les établissements de soins de santé et les établissements d'enseignement postsecondaire. Un tel résultat porte à croire que la population de la communauté avoisinante était adéquatement immunisée malgré les taux de vaccination sous-optimaux établis à des moments précis.

La relation de travail productive qui avait été établie au préalable entre l'autorité locale de santé publique et les dirigeants des communautés néerlandaises réformées a grandement facilité l'intervention à la suite de l'éclosion. Cette relation s'est révélée inestimable, car elle a permis de bâtir un lien de confiance avec la communauté avant l'éclosion. Le fait de faire appel à une infirmière liée à la communauté a renforcé davantage la communication et le degré d'aisance entre la communauté et les autorités sanitaires. Cette relation a permis à la communauté de participer activement au contrôle de la propagation de l'éclosion tout en préservant ses pratiques religieuses. La déclaration rapide des cas et l'observance et l'exécution des mesures de santé publique par la communauté, en particulier les restrictions de voyage pendant la période cruciale de la semaine de relâche, ont évité la propagation de la rougeole vers des régions plus peuplées où une retransmission aurait pu survenir.

Les communautés confessionnelles non vaccinées demeurent vulnérables aux maladies évitables par la vaccination. Des communautés de ce genre ont subi des éclosions épisodiques de polio, de rougeole, de rubéole et d'oreillons attribuables à une transmission par les frontières internationales en raison de voyages fréquents pour rendre visite à des parents et amis de communautés associées (13-17). Les bureaux de santé publique locaux qui desservent de telles communautés augmentent leurs capacités et échangent entre eux les apprentissages tirés de leurs expériences pour adapter leurs interventions et optimiser le contrôle des éclosions dans ces populations.

\section{Remerciements}

Un grand nombre de membres du personnel de la Fraser Health Authority ont participé à la collecte de données pendant l'éclosion. Le laboratoire de santé publique et de référence en microbiologie de la Colombie-Britannique a réalisé tous les tests diagnostiques pour la rougeole et le Laboratoire national de microbiologie, de l'Agence de la santé publique du Canada, s'est occupé des tests de confirmation et du génotypage.

\section{Conflit d'intérêts}

Aucun

\section{Références}

(1) King A, Varughese P, De Serres G, Tipples GA, Waters J. Working Group on Measles Elimination. Measles elimination in Canada. J Infect Dis. 2004 May 1;189 Suppl 1:S236-42.

(2) BC Centre for Disease Control. Communicable disease control manual, Chapter 2: Immunization, Section IA, Introduction. 11.0 History of immunization in BC. Page 31; May 2009. http://www.bccdc.ca/NR/rdonlyres/EB07BC36BF2D-47A6-BF44-DC286F762E9F/0/SectionIA_IntroductionJune2014.pdf.

(3) Public Health Agency of Canada. Elimination of measles, rubella and congenital rubella syndrome in Canada: Documentation and verification report. Submitted to the Pan American Health Organization/ World Health Organization. Ottawa ON: PHAC; December 2011. Executive summary available at: http://www.phacaspc.gc.ca/im/vpd-mev/measles-rougeole-mrer-eng.php.

Disponible en français: http://www.phac-aspc.gc.ca/im/vpd-mev/measles-rougeole-mg-fra.php

(4) BC Centre for Disease Control. British Columbia annual summary of reportable diseases 2010. Measles. Page 20. Vancouver BC : BCCDC; 2012. http://www.bccdc.ca/NR/rdonlyres/6F0D23A6-18E8-4983-AE53A7F0C7F0D91B/0/2010CDAnnualReportFinal.pdf.

(5) Gardy JL, Naus M, Amlani A, Chung W, Kim H, Tan M, Severini A, Krajden M, Puddicombe D, Sahni V, Hayden AS, Gustafson R, Henry B, Tang P. Whole-genome sequencing of $\mathrm{H} 1$ and D8 genotype measles outbreaks following the Olympic Winter Games reveals viral transmission routes. 2015. J. Infect. Dis. In press.

(6) Knol M, Urbanus A, Swart E, Mollema L, Ruijs W, van Binnendijk R, Te Wierik M, de Melker H, Timen A, Hahné S. Large ongoing measles outbreak in a religious community in the Netherlands since May 2013. Euro Surveill. 2013 Sep 5;18(36):pii=20580. http://eurosurveillance.org/ViewArticle.aspx?Articleld=20580. 
(7) Netherlands National Institute for Public Health and the Environment. Ministry of Health, Welfare and Sport [Internet]. Measles. Bilthoven NL: RIVM; 2015. http://www.rivm.nl/en/Topics/M/Measles.

(8) Vivian Suttorp. Presentation at the Western Canada Immunization Forum, March 2014. Thursday March 6th, Day 2, Morning Plenary. http://www.health.alberta.ca/professionals/Western-Canada-Immunization-Forum.html.

(9) Public Health Act. Health Act Communicable Disease Regulation. B.C. Reg. 4/84 O.C. 6/83 http://www.bclaws.ca/.

(10) British Columbia Centre for Disease Control. Measles case definition.; 2014. http://www.bccdc.ca/dis-cond/az/ m/Measles/Measlecase.htm.

(11) $\quad B \bar{C}$ Centre for Disease Control. Measles Case Report Form.; 2014. http://www.bccdc.ca/NR/rdonlyres/2AD4360A9AB4-440B-B522-5A96046D6250/0/Measles_ProvSurveillanceForm_20140918.pdf.

(12) BC Centre for Disease Control. Communicable disease control. Chapter I: Management of specific diseases. Measles.; 2015. http://www.bccdc.ca/dis-cond/comm-manual/CDManualChap1.htm.

(13) Ofner M, Naus M, Martin E, Stolk B. Community surveillance for wild poliovirus in Ontario, 1993. CCDR. 1994;20:214-216. Reprinted in CMAJ. 1995;152(12):1997-1998.

(14) van Steenbergen JE, van den Hof S, Langendam MW, van de Kerkhof JHTC, Ruijs WLM. Measles outbreak Netherlands, April 1999 - January 2000. MMWR. 2000;49(14):299-303. http://www.cdc.gov/mmwr/preview/mmwrhtml/mm4914a2.htm.

(15) Jansen VA, Stollenwerk N, Jensen HJ, Ramsay ME, Edmunds WJ, Rhodes CJ. Measles outbreaks in a population with declining vaccine uptake. Science. 2003;301:804; PMID:12907792. http://dx.doi.org/10.1126/science.1086726.

(16) Hahné S, Macey J, van Binnendijk R, Kohl R, Dolman S, van der Veen Y, et al. Rubella outbreak in the Netherlands, 2004-2005: High burden of congenital infection and spread to Canada. Pediatr Infect Dis J. 2009;28:795-800; PMID:19710586. http://dx.doi.org/10.1097/INF.0b013e3181a3e2d5.

(17) Wielders CC, van Binnendijk RS, Snijders BE, Tipples GA, Cremer J, Fanoy E, Dolman S, Ruijs WI, Boot HJ, de Melker HE, Hahné SJ. Mumps epidemic in orthodox religious low-vaccination communities in the Netherlands and Canada, 2007 to 2009. Euro Surveill. 2011 Oct 13;16(41). pii: 19989. 